SCIENTIFIC LETTER

\title{
Echocardiography in differentiating tuberculous from chronic idiopathic pericardial effusion
}

\author{
S George, A L Salama, B Uthaman, G Cherian
}

Heart 2004;90:1338-1339. doi: 10.1136/hrt.2003.020081

$\mathrm{T}$ uberculous pericarditis continues to have a high incidence in developing countries. Despite effective antimicrobial treatment and improved public health in developed countries, there has been a recent surge in the incidence of both atypical and classical tuberculosis in immunocompromised patients, especially those infected with HIV.

Only a few studies have been published describing the echocardiographic features of tuberculous pericardial effusion, especially with reference to echocardiographic intrapericardial abnormalities. ${ }^{1-5}$

The aim of this study was to evaluate the echocardiographic features of tuberculous pericardial effusion and assess the diagnostic and prognostic usefulness of echocardiographic intrapericardial abnormalities in distinguishing between patients with tuberculous pericardial effusion and idiopathic/viral pericardial effusion, which has not been previously studied.

\section{METHODS}

We analysed the medical records and echocardiograms of 27 consecutive patients with a discharge diagnosis of tuberculous pericardial effusion (group A), and 15 consecutive patients with a diagnosis of viral/idiopathic pericardial effusion (group B). A complete clinical history, physical examination, ECG, chest $x$ ray and routine blood tests were performed in all patients. All patients underwent pericardiocentesis and three had surgical pericardiectomy. Pericardial biopsies were obtained at the time of pericardiocentesis. The pericardial fluid and biopsy specimens were sent for cytology, histopathology examination, acid fast bacilli staining, and culture for Mycobacterium tuberculosis.

The diagnosis of tuberculous pericarditis was based on either a positive $M$ tuberculosis culture from the pericardial fluid or pericardial biopsy, detection of acid fast bacilli or a typical granuloma on histopathology, or the presence of pulmonary tuberculosis.

The diagnosis of viral pericarditis was based on antibody studies. Viral and idiopathic pericardial effusions were grouped together for analysis as chronic idiopathic pericardial effusion.

All patients underwent computed tomography (CT) of the thorax for assessment of the pericardium. Echocardiography was performed with a 2.5/3.0 MHz transducer on an Acuson Sequoia 512 realtime ultrasound imaging system. The echocardiographic images were recorded on videotape and analysed retrospectively for the following: pericardial thickening, defined as the thickest part of the pericardium measuring more than $2 \mathrm{~mm}$ on two dimensional echocardiography; pericardial effusion, estimated as mild, moderate, or large according to criteria proposed by Eisenberg and colleagues $^{6}$; exudative coating/deposits, defined as shaggy echo dense images with a mass like appearance surrounding the epicardium; fibrin strands, defined as multiple linear or band like structures from the epicardium or pericardium protruding or crossing the pericardial space. ${ }^{5}$

The clinical characteristics and frequency of echocardiographic abnormalities were compared between group A (tuberculous pericarditis) and group B (chronic idiopathic pericardial effusion) using the two tailed $t$ test. A probability value of $\mathrm{p}<0.05$ was considered significant.

\section{RESULTS}

In group A $(n=27)$ there were $61.5 \%$ male and $38.5 \%$ female patients. The mean (SD) age was 36 (12) years in group A and 45 (19) years in group B. All 27 patients in group A underwent pericardiocentesis and three patients underwent pericardiectomy. All patients in group B underwent pericardiocentesis and none required pericardiectomy. The diagnosis of tuberculous pericarditis was established by a positive culture for $M$ tuberculosis in 8/27 patients (33.3\%) and a positive pericardial biopsy for caseating granuloma in 24/27 patients. Three patients had pulmonary tuberculosis and all patients responded to specific antituberculous therapy.

All patients in group A had a large pericardial effusion of at least $20 \mathrm{~mm}$. The average size of the effusion was 32 (6) $\mathrm{mm}$ in group A $v 27$ (4) $\mathrm{mm}$ in group B. Thickened pericardium was found in 20/27 patients (70.8\%) in group A and 2/15 patients $(13.3 \%)$ in group B. The mean pericardial thickness was significantly more in group A $(4.3$ (1.8) mm $v 2.1$ $(0.35) \mathrm{mm}, \mathrm{p}<0.05)$. The echocardiographic incidence of cardiac tamponade was higher in group A $169 \% \vee 50 \%$, $p<0.05)$. The majority of patients had a generalised effusion and only two patients had a loculated effusion in the tuberculous pericarditis group. Intrapericardial exudates were more frequent $(96 \% \vee 46.6 \%, \mathrm{p}<0.001)$ and thicker in group A (4.5 (2) mm $v 2.8(0.69) \mathrm{mm}, \mathrm{p}<0.013)$. Fibrin strands were present more often in group A $(66 \% v 13.3 \%$, $\mathrm{p}<0.003)$. Two patients presented with features of effusive constrictive pericarditis at the initial echocardiogram (fig l). Two patients developed effusive constrictive pericarditis on follow up.

\section{DISCUSSION}

Tuberculous pericarditis continues to be a problem in both developed and developing countries where viral or idiopathic pericardial effusion is another commonly encountered cause of pericardial effusion.

There are only a few studies describing the echo features of tuberculous pericarditis; our study is the only one that evaluates the diagnostic utility of two dimensional echocardiography in differentiating tuberculous pericarditis from chronic idiopathic pericardial effusion with special emphasis on intrapericardial abnormalities.

Band-like intrapericardial echoes and masses were initially described in the pericardial cavity in patients with pericardial effusion of varying aetiology, most of which were associated with malignancy. ${ }^{2}{ }^{3}$ 


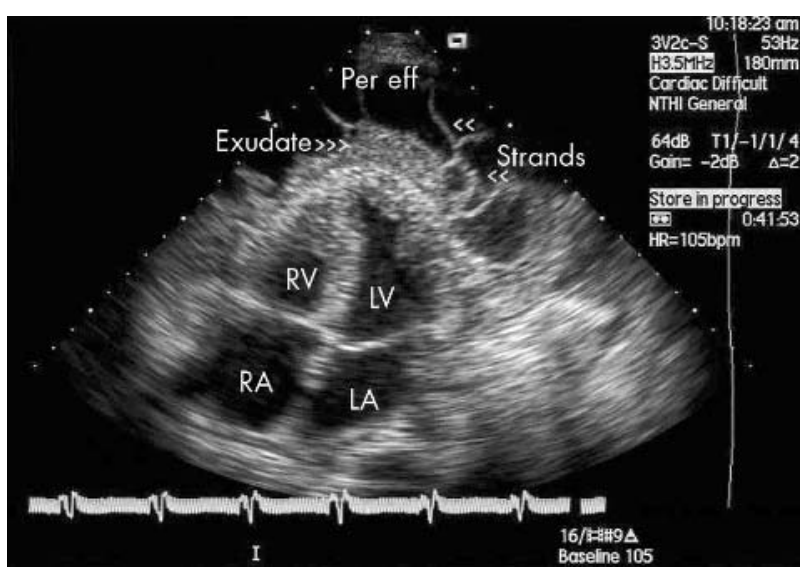

Figure 1 Apical four chamber view of a two dimensional echocardiogram of a patient with tuberculous pericardial effusion showing multiple fibrin strands as linear or band like structures crossing the pericardial space or protruding from the epicardium or parietal pericardium and exudates. LA, left atrium; LV, left ventricle; Per eff, pericardial effusion; RA, right atrium; RV, right ventricle.

The first report of intrapericardial abnormalities associated with tuberculous pericardial effusion was probably by Chia and colleagues who described linear frond-like echo dense structures protruding into the pericardial cavity forming a dense mass as a result of fibrinous pericarditis. ${ }^{4}$

Liu and colleagues demonstrated that thickened pericardium and fibrin strands were highly specific (94\% and $88 \%$, respectively), and exudative coating had a high sensitivity $(100 \%)$, but low specificity $(22 \%)$, in the diagnosis of tuberculous pericarditis. Their series had only one patient of chronic idiopathic pericardial effusion. ${ }^{5}$

In this study we found that intrapericardial abnormalities such as fibrin strands, exudative coating, and deposits, were more common in tuberculous pericardial effusion compared to chronic idiopathic pericardial effusion. Earlier studies had not specifically addressed the issue of differentiating these two forms of pericardial effusion by echocardiography. We also measured the thickness of the exudative coating/deposits by echocardiography and this was found to be 4.5 (2.0) $\mathrm{mm}$ in tuberculous pericardial effusion and $2.8(0.69) \mathrm{mm}$ in chronic idiopathic pericardial effusion $(\mathrm{p}<0.013)$. This is useful as in our study $47 \%$ of patients with chronic idiopathic pericardial effusion demonstrated exudates on echocardiography. These exudates are probably caused by non-specific fibrinous changes.

Echocardiographic evidence of cardiac tamponade was more common in tuberculous pericardial effusion (19/27 patients in group A $v 6 / 15$ patients in group B, p < 0.05). Two patients from group A developed effusive constrictive pericarditis on follow up and two patients presented with effusive constrictive pericarditis. None of the patients in group $\mathrm{B}$ had developed constriction at a mean follow up period of 16 (11) months. Earlier studies have suggested that intrapericardial abnormalities such as strands and thick exudates that are caused by fibrin may organise and later lead to features of constrictive pericarditis. ${ }^{1}$ This, however, has not been our experience as 24/27 patients were cured at follow up with a normal pericardium on chest CT studies.

Intrapericardial echo abnormalities such as a greater degree of pericardial thickening, frequency and thickness of exudative coating or deposits, and strands crossing the pericardial space, are useful in the diagnosis of tuberculous pericardial effusion and in differentiating this entity from chronic idiopathic pericardial effusion.

\section{Authors' affiliations}

S George, A L Salama, Department of Cardiology, Chest Disease Hospital, Kuwait

B Uthaman, G Cherian, Department of Medicine, Faculty of Medicine, Kuwait University

Correspondence to: Dr Susan George, PO Box 1602, Ardhya 92400, Kuwait; samuelsusan@yahoo.com

Accepted 13 January 2004

\section{REFERENCES}

1 Alio-Bosch J, Candell-Riera J, Monge-Rangel L, et al. Intrapericardial echocardiographic images and cardiac constriction. Am Heart J 1991;208:121-207

2 Martin RP, Bowden R, Filly K, et al. Intrapericardial abnormalities patients with pericardial effusion. Circulation 1980;61:568-72.

3 Chandraratna PAN, Aronow W. Detection of pericardial metastases by cross sectional echocardiography. Circulation 1981;63:197-8.

4 Chia BL, Choo M, Tan A, et al. Echocardiographic abnormalities in tuberculous pericardial effusion. Am Heart J 1984;107:1034-5.

5 Liu PY, Li YH, Tsai WC, et al. Usefulness of echocardiographic intrapericardial abnormalities in the diagnosis of tuberculous pericardial effusion. Am J Cardiol 2001;87:1133-5.

6 Eisenberg MJ, Oken K, Guerrero S, et al. Prognostic value of echocardiography in hospitalized patients with pericardial effusion. Am J Cardiol 1992;70:934-9. 